Communications in Physics, Vol. 31, No. 2 (2021), pp. 169-178

DOI:10.15625/0868-3166/15564

\title{
SIMULATION STUDY ON SUPERCONTINUUM GENERATION AT NORMAL DISPERSION REGIME OF A CARBON DISULFIDE-CORE PHOTONIC CRYSTAL FIBER
}

\author{
BIEN CHU VAN ${ }^{1}$, DINH QUANG HO ${ }^{2}$, LE THI HA ${ }^{1}$, VAN CAO LONG ${ }^{3}$, VU VAN HUNG ${ }^{4}$ \\ AND HIEU LE VAN ${ }^{1, \dagger}$ \\ ${ }^{1}$ Faculty of Natural Sciences, Hong Duc University, 565 Quang Trung Street, Thanh Hoa City, \\ Vietnam \\ ${ }^{2}$ School of Chemistry, Biology and Environment, Vinh University, 182 Le Duan Street, Vinh City, \\ Nghe An Province, Vietnam \\ ${ }^{3}$ Institute of Physics, University of Zielona Gora, Prof. Szafrana 4a, 65-516 Zielona Gora, Poland \\ ${ }^{4}$ Office of Thanh Hoa People's Committee, 35 Le Loi Street, Thanh Hoa City, Thanh Hoa \\ Province, Vietnam \\ E-mail: ${ }^{\dagger}$ levanhieu@hdu.edu.vn \\ Received 7 October 2020 \\ Accepted for publication 17 November 2020 \\ Published 15 April 2021
}

\begin{abstract}
A photonic crystal fiber with a hollow core filled with carbon disulfide $\left(C S_{2}\right)$ is proposed as a new source of supercontinuum light. We numerically study guiding properties of modeled fibers including the dispersion and the effective mode area of the fundamental mode. As a result, octave spanning of the SC spectrum was achieved in the wavelength range of near-IR from 1.25 $\mu \mathrm{m}$ to $2.3 \mu \mathrm{m}$ with $90 \mathrm{fs}$ pulse and energy of $1.5 \mathrm{~nJ}$ at a pump wavelength of $1.55 \mu \mathrm{m}$. The proposed fibers are fully compatible with all-silica fiber systems, in particular, could be used for all-fiber SC sources and new low-cost all-fiber optical systems.
\end{abstract}

Keywords: nonlinear optics, photonic crystal fiber, liquid, supercontinuum generation.

Classification numbers: 42.65.Jx; 42.55.Tv; 77.84.Nh; 88.60.np.

\section{INTRODUCTION}

Photonic crystal fibers (PCFs), also known as micro-structured fibers or holey fibers have been a considerably attractive topic for optical community all over the world for the past decades. (C)2021 Vietnam Academy of Science and Technology 
PCFs can be used in a variety of research fields and practical applications. Here it is worth mentioning about fiber light sources, supercontinuum generation devices, fiber optic sensors or nonlinear devices [1,2], endless single-mode properties [3], single-polarization single-mode operation [4], high birefringence [5], tailorable dispersion profiles and flat or ultra-flat dispersion [6,7]. Among others, the generation of supercontinuum (SC) is one of the most important applications. Because of its unique properties, SC generation has been exploited in various prospects including optical coherence tomography, optical metrology, multimodal bio-photonic imaging, and highspeed optical communication [8-11].

The SC generation occurs when the ultra-short optical pulses are pumped into a highly nonlinear medium [12]. It is a complex process of spectral broadening of ultra-short optical pulses such as femtosecond or picosecond, which undergo a number of nonlinear interactions in/with the optical nonlinear medium, such as modulation instability, self-phase-modulation, four-wavemixing [13-15] in the normal dispersion region or stimulated Raman scattering, self-steepening and soliton fission $[16,17]$ in the anomalous dispersion region.

In order to get an efficient broadband SC generation, PCFs with flat dispersion characteristic and highly nonlinear glass is usually utilized. In this manner, the PCFs are usually made of silica or highly nonlinear glasses $[11,18,19]$. Silica fibers might efficiently create SC spectra in the visible to the near-infrared (NIR) range [11]. Meanwhile, PCFs made of highly nonlinear glasses offer a higher nonlinear refractive index in comparison with silica as well as have a broadband transmission until the mid-IR range. Thus, it allows the SC generation in the mid-IR range [18,19]. However, the SC generation sources of PCFs from non-silica with highly nonlinear solid core suffer from high costs and a complex fabrication process.

Recently, liquid-core PCFs have been practically demonstrated as a considerable useful replacement for obtaining efficient spectral broadening. In this way, several nonlinear liquids, named a few, carbon disulfide $\left(\mathrm{CS}_{2}\right)$, ethanol $\left(\mathrm{C}_{2} \mathrm{H}_{5} \mathrm{OH}\right)$, carbon tetrachloride $\left(\mathrm{CCl}_{4}\right)$, chloroform $\left(\mathrm{CHCl}_{3}\right)$, toluene $\left(\mathrm{C}_{7} \mathrm{H}_{8}\right)$ and nitrobenzene $\left(\mathrm{C}_{6} \mathrm{H}_{5} \mathrm{NO}_{2}\right)$ have been used for this approach [20-25]. The results indicated that the generated SC spectra can be controlled by changing temperature, pressure or applying an electric field. Moreover, the SC spectrum can be achieved in both the normal and anomalous dispersion regimes with high coherence [26]. It can be explained that the liquids have higher nonlinear refractive indices than those for solids. In detail, this leads to the appearance of interesting nonlinear phenomena, especially, generated SC having a lower peak power than for solid fiber [21]. Although the SC generation in liquid-infiltrated PCFs has been widely studied, their performance in terms of spectral flatness, bandwidth, and the coherent SC spectrum still need further optimization.

In this paper, we proposed a PCF based on fused silica glass with the core filled carbon disulfide $\left(\mathrm{CS}_{2}\right)$. We have selected $\mathrm{CS}_{2}$ because it has high nonlinear refractive index $n_{2}=$ $3.2 \times 10^{-19} \mathrm{~m}^{2} / \mathrm{W}$ at $\lambda=1550 \mathrm{~nm}$ [27] (approximately eleven-time higher than for fused silica $\left.n_{2}=2.79 \times 10^{-20} \mathrm{~m}^{2} / \mathrm{W}[28]\right)$. Beside that $\mathrm{CS}_{2}$ provides a super-wide transparent window from the visible to mid-IR (up to $12 \mu \mathrm{m}$ ) [29]. Here, we present the optimization of the dispersion properties by modifying photonic fiber structure parameters, i.e., lattice constant and size of air hole to obtain all-normal-flat-dispersion and the maximum dispersion is the closest value to the horizontal axis. And the SC generation in the optimized PCF is theoretically demonstrated by using generalized nonlinear Schrödinger equation (GNLSE). In addition, the discussions about the advantages of this fiber are presented. 


\section{DESIGN OF THE PCF AND THEORETICAL MODEL}

In this study, we design and optimize fiber structures by the finite element method which is widely used for analyzing the optical waveguide. Numerical analysis was performed using the Lumerical Mode Solution software [30]. The cross-section of the proposed PCF is shown in Fig. 1. The geometry of the PCF is a standard hexagon with eight rings of air-holes, surrounding an empty core is filled with carbon disulfide $\left(\mathrm{CS}_{2}\right.$-core) in the fused silica cladding. The filling factor of the cladding is defined as $f=d / \Lambda$, where $d$ is the diameter of air hole and $\Lambda$ is the lattice constant. The diameter of $\mathrm{CS}_{2}$-core is bigger and fulfills the relation $D_{c}=2 . \Lambda-1.2 d$, where $D_{C}$ is the diameter of $\mathrm{CS}_{2}$-core.

The refractive index characteristics are simulated using the Sellmeier formula as shown below:

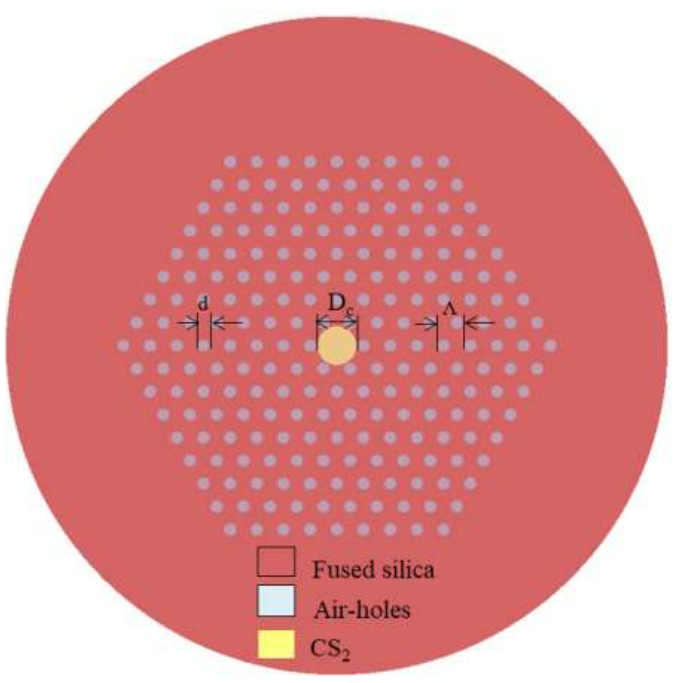

Fig. 1. Schematic of the proposed PCF, where $D_{\mathrm{c}}$ is the diameter of the $\mathrm{CS}_{2}$-core.

$$
n(\lambda)=\sqrt{1+\frac{B_{1} \lambda^{2}}{\lambda^{2}-C_{1}}+\frac{B_{2} \lambda^{2}}{\lambda^{2}-C_{2}}+\frac{B_{3} \lambda^{2}}{\lambda^{2}-C_{3}},}
$$

where, the $B_{\mathrm{i}}$ and $C_{\mathrm{i}}\left(\mu \mathrm{m}^{2}\right)$ are Sellmeier's coefficients as given in Table 1 .

Table 1. The Sellmeier's coefficients of fused silica and $\mathrm{CS}_{2}[31,32]$.

\begin{tabular}{cl}
\hline Sellmeier's coefficients & Values \\
\hline Fused silica & \\
$\mathrm{B}_{1}$ & 0.6694226 \\
$\mathrm{~B}_{2}$ & 0.4345839 \\
$\mathrm{~B}_{3}$ & 0.8716947 \\
$\mathrm{C}_{1}$ & $4.4801 \times 10^{-3} \mu \mathrm{m}^{2}$ \\
$\mathrm{C}_{2}$ & $1.3285 \times 10^{-2} \mu \mathrm{m}^{2}$ \\
$\mathrm{C}_{3}$ & $95.341482 \mu \mathrm{m}^{2}$ \\
Carbon disulfide & \\
$\mathrm{B}_{1}$ & 1.50387 \\
$\mathrm{C}_{1}$ & $3.049 \times 10^{-2} \mu \mathrm{m}^{2}$ \\
\hline
\end{tabular}

The material dispersion is directly included in the calculations through the three-term Sellmeier equation. The chromatic dispersion D $(\lambda)$ of a PCF is easily calculated from the $n_{\text {eff }}$ values 
versus the wavelength using the following formula [33]:

$$
D(\lambda)=-\frac{\lambda}{c} \frac{d^{2} \operatorname{Re}\left[n_{e f f}\right]}{d \lambda^{2}}
$$

where $\operatorname{Re}\left[\mathrm{n}_{\text {eff }}\right]$ is the real part of the refractive index, $\lambda$ is the operating wavelength, and $c$ is the velocity of light in a vacuum.

The effective area $A_{\text {eff }}$ is a qualitative measurement of the cross section area covered by guided mode of the fiber and is calculated as follows [33]:

$$
A_{e f f}=\frac{\left(\iint|E|^{2} d x d y\right)^{2}}{\iint|E|^{4} d x d y},
$$

where $E$ is the electric field in the medium obtained by solving an eigenvalue problem derived from Maxwell's equations.

Meanwhile, based on the effective mode area we can obtain another important parameter for optical communication which is the nonlinearity of the fiber. The nonlinear coefficient of the PCF can be defined as [33]:

$$
\gamma(\lambda)=\frac{2 \pi n_{2}}{\lambda A_{e f f}}
$$

where $n_{2}$ is the nonlinear coefficient.

\section{OPTIMIZATION OF ALL NORMAL DISPERSION PROPERTIES FOR OPTICAL

\begin{tabular}{|c|c|c|c|c|}
\hline \multirow{2}{*}{$d / \Lambda$} & \multicolumn{3}{|c|}{$\Lambda(\mu \mathbf{m})$} & \multirow[b]{2}{*}{2.5} \\
\hline & 1.0 & 1.5 & 2.0 & \\
\hline 0.20 & 0.88 & 1.32 & 1.76 & 2.20 \\
\hline 0.25 & 0.85 & 1.28 & 1.70 & 2.13 \\
\hline 0.30 & 0.82 & 1.23 & 1.64 & 2.05 \\
\hline 0.35 & 0.79 & 1.19 & 1.58 & 1.98 \\
\hline 0.40 & 0.76 & 1.14 & 1.52 & 1.90 \\
\hline 0.45 & 0.73 & 1.10 & 1.46 & 1.83 \\
\hline 0.50 & 0.70 & 1.05 & 1.40 & 1.75 \\
\hline 0.55 & 0.67 & 1.01 & 1.34 & 1.68 \\
\hline 0.60 & 0.64 & 0.96 & 1.28 & 1.60 \\
\hline 0.65 & 0.61 & 0.91 & 1.22 & 1.53 \\
\hline 0.70 & 0.58 & 0.87 & 1.16 & 1.45 \\
\hline 0.75 & 0.55 & 0.82 & 1.10 & 1.38 \\
\hline 0.80 & 0.52 & 0.78 & 1.04 & 1.30 \\
\hline
\end{tabular} FIBER WITH VARIOUS SIZES}

Table 2. The $\mathrm{CS}_{2}$-core diameter of the designed PCF. 


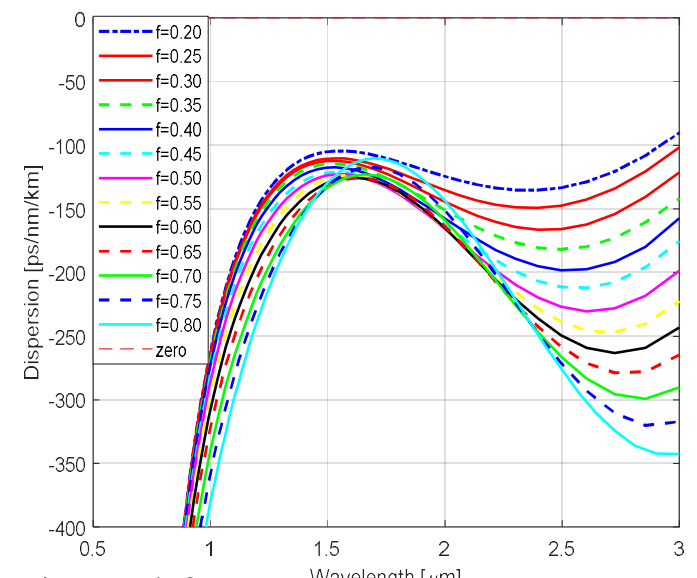

a) $\Lambda=1.0 \mu \mathrm{m}$



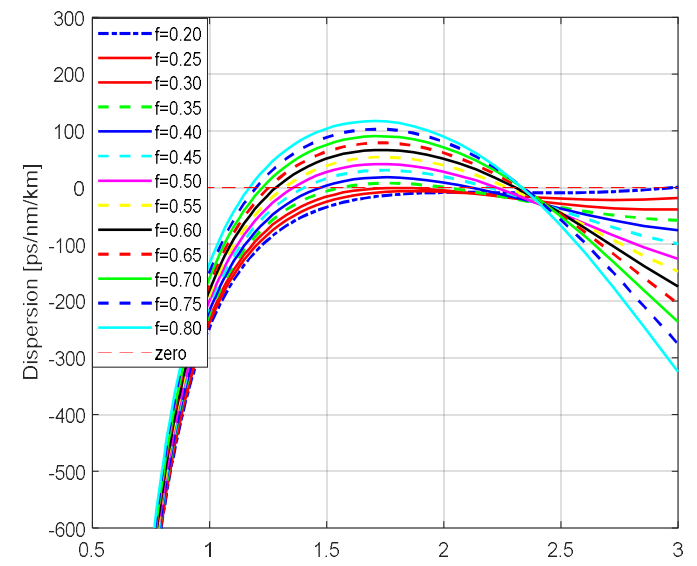

b) $\Lambda=1.5 \mu \mathrm{m} \quad$ Wavelength $[\mu \mathrm{m}]$

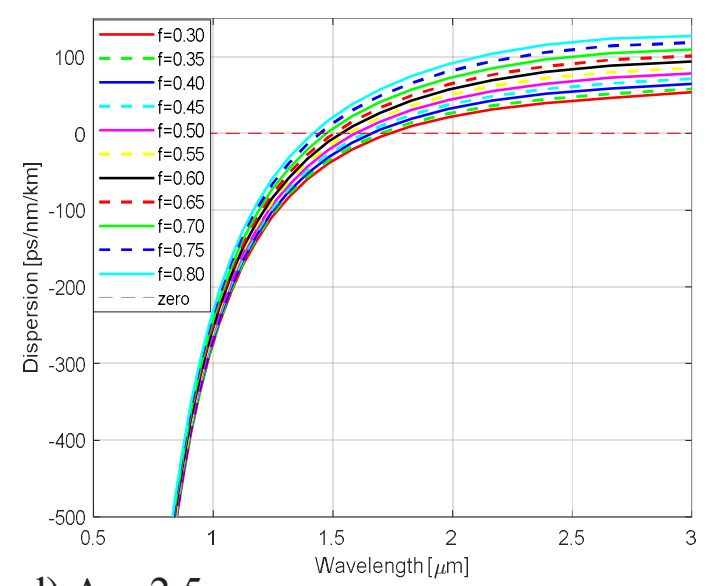

d) $\Lambda=2.5 \mu \mathrm{m}$

Fig. 2. Characteristics of the PCF mode dispersion for filling factor $\mathrm{f}$ values in the range from 0.20 to 0.80 and lattice constants (a) $1.0 \mu \mathrm{m}$, (b) $1.5 \mu \mathrm{m}$, (c) $2.0 \mu \mathrm{m}$, and (d) $2.5 \mu \mathrm{m}$.

The optimization criteria aimed at the generation of SC in all normal dispersion regimes with pumping at $1.55 \mu \mathrm{m}$, followed the flatness, sign of the dispersion charactericties and distance from ZDW if located in the analyzed wavelength range. Looking for the optimal structure of a PCF, we consider the structure with the lattice constants changing from $1.0 \mu \mathrm{m}$ to $2.5 \mu \mathrm{m}$ with step of 0.5 and the linear filling factors varied from 0.20 to 0.80 with step of 0.05 . The smallest $\mathrm{CS}_{2}$-core diameter was $0.53 \mu \mathrm{m}$ for $\Lambda=1.0 \mu \mathrm{m}$ and $f=0.8$. The biggest $\mathrm{CS}_{2}$-core diameter diameter was $2.20 \mu \mathrm{m}$ for $\Lambda=2.5 \mu \mathrm{m}$ and $f=0.2$. For more details of the $\mathrm{CS}_{2}$-core diameters are illustrated in Table 2.

Normally, the first ring of air-holes surrounding the core strongly affects the dispersion properties of PCF as well as the zero-dispersion wavelength (ZDW), while the outer rings play the role in the mode attenuation, especially for higher modes [26]. In the simulation, we used a constant filling factor for all rings of PCF to simplify the development of this fiber in the future. 
On the other hand, we do not also check if the modelled fiber is a single-mode fiber or multi-mode fiber. This step is only performed for optimized fibers.

Figure 2 shows that the dispersion characteristics of $\mathrm{CS}_{2}$-core PCF can be tuned by changing the linear filling factor and lattice constant. We observe that all $\mathrm{CS}_{2}$-core PCFs have flat dispersion characteristics in the considered range.

For a given $\Lambda(\Lambda=1.0 \mu \mathrm{m})$, the dispersion characteristics are allnormal dispersion in the full range wavelength. In the case of $\Lambda=$ $1.5 \mu \mathrm{m}$, the dispersion characteristics exist only in the normal dispersion regime or a part exist both regimes. On the contrary, the maximum dispersion is greater than zero, which means there is both normal and anomalous dispersion.

Moreover, for a given $f$ value, the dispersion characteristics and ZDWs are shifted toward longer waves and flattened with increasing $\Lambda$. Meanwhile, for a given $\Lambda$ value, $\mathrm{ZDW}$ is usually shifted toward longer waves with decreasing $f$.

On the basis of initial numerical investigations, we choose the PCF structure with following parameters: $\Lambda=1.5 \mu \mathrm{m}$ and $f=0.30$. The numerical calculations of dispersion characteristics of the optimal structure are presented in Fig. 3. This fiber has optimum dispersion characteristics since this fiber has all normal dispersion and the dispersion curve has achieved flatness. In addition, the dispersion at the pump wavelength equal $-8.1 \mathrm{ps} / \mathrm{nm} / \mathrm{km}$, which is the closest to the zero for all lattice constants which can obtained

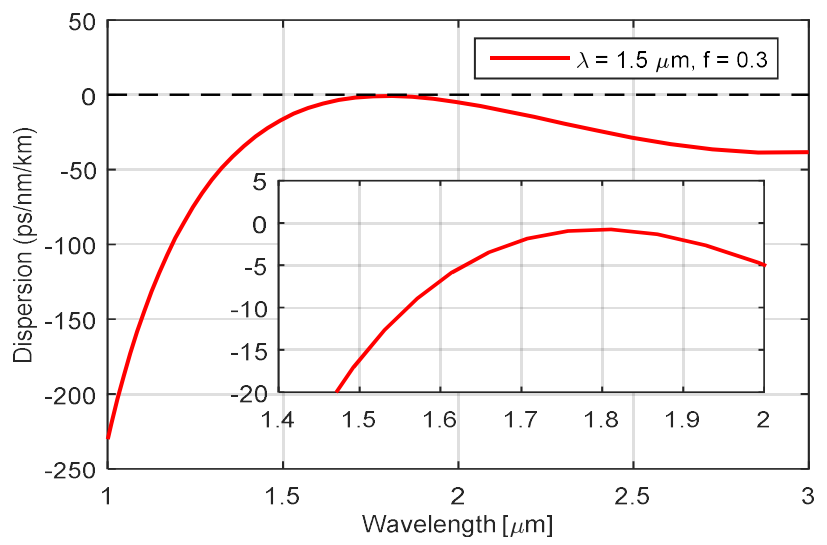

Fig. 3. Numerical calculations of dispersion characteristics in $\mathrm{CS}_{2}$-filled core optimal fiber structure.

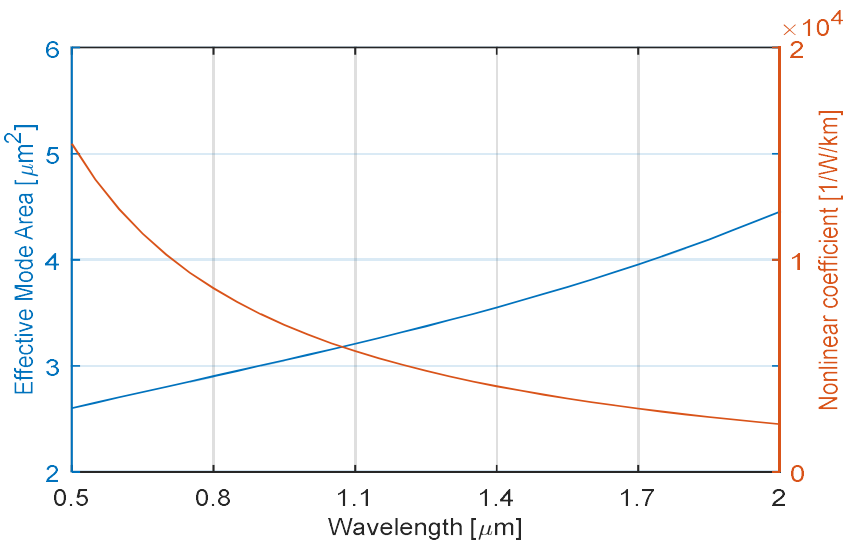

Fig. 4. Calculations of effective mode area and the nonlinear coefficient of the PCF infiltrated with $\mathrm{CS}_{2}$. all normal dispersion region.

Figure 4 presents the effective mode area and nonlinear coefficients of the optimal fiber. In this case, because the core diameter is relatively small, it leads also to smallness in the effective mode area of the optimal structure. In addition, the modal area of the fundamental mode increases linearly with the wavelength. For the wavelength of $0.5 \mu \mathrm{m}$ the modal area equals $2.60 \mu \mathrm{m}^{2}$, while 
for the wavelength of $2.0 \mu \mathrm{m}$, the modal area equals $4.11 \mu \mathrm{m}^{2}$. Thus, the mode area is not changed much within the wavelength range.

\section{SUPERCONTINUUM GENERATION IN THE OPTIMAL STRUCTURE}

The SC generation of the optimized PCF structure was simulated by numerically solving of the generalized nonlinear Schrödinger equation (GNLSE) when the split-step Fourier method is used [33]:

$$
\begin{aligned}
\frac{\partial A}{\partial z}= & -\frac{\alpha}{2} A+\sum_{n \geq 2} \beta_{n} \frac{i^{n+1}}{n !} \frac{\partial^{n}}{\partial T^{n}} A \\
& +i \gamma \frac{1}{\omega_{0}}\left(1+\frac{\partial}{\partial T}\right)\left[\left(1-f_{R}\right)|A|^{2} A+f_{R} A \int_{0}^{\infty} h_{R}(t)|A(z, T-t)|^{2} d t\right]
\end{aligned}
$$

where, $A=A(z, t)$ is the complex amplitude of the optical field, $\alpha$ is the total loss in the PCF, $\beta_{n}$ are the dispersion coefficients associated with the Taylor series expansion, $\gamma$ is the nonlinear coefficient, $\lambda_{c}$ is the central wavelength, $f_{R}$ is the Raman fraction response to nonlinear polarization, $h_{R}(t)$ represents the Raman response function which is given by [33]:

$$
h_{R}(t)=\left(\tau_{1}^{2}+\tau_{2}^{2}\right) \tau_{1}^{-1} \tau_{2}^{-2} \exp \left(-t / \tau_{2}\right) \sin \left(-t / \tau_{1}\right) .
$$

In simulations, the following parameters were used: the fiber length $20 \mathrm{~cm}$, the Gaussianshape pulse of duration 90 fs and the Raman fraction $f_{R}=0.89, \tau_{1}=1.68 \mathrm{ps}, \tau_{2}=0.14 \mathrm{ps}$ [27], the nonlinear refractive index of $\mathrm{CS}_{2} n_{2}=3.2 \times 10^{-19} \mathrm{~m}^{2} \mathrm{~W}^{-1}$ [27] and the pump wavelength of $1.55 \mu \mathrm{m}$.
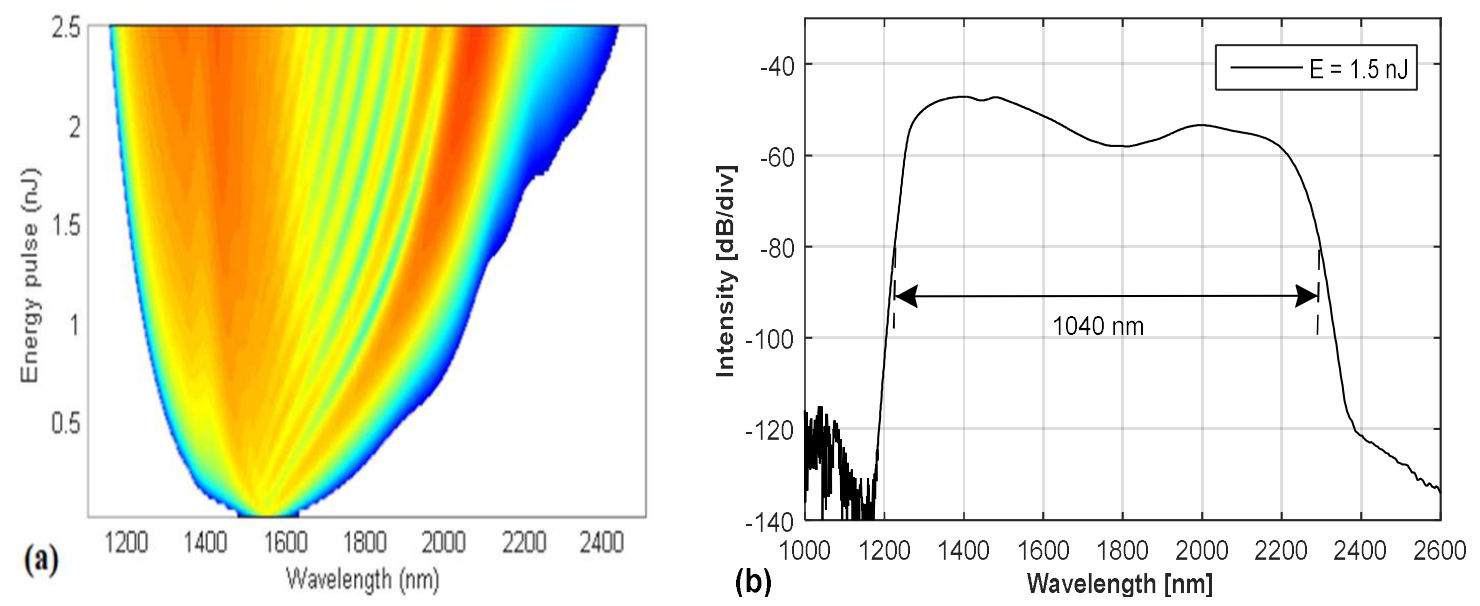

Fig. 5. Spectral intensity of PCF with various energies.

Fig. 5(a) presents the evolution of the broadened spectra as a function of input energy at the length $20 \mathrm{~cm}$. In the case of input pulse energy are smaller than $0.5 \mathrm{~nJ}$, the initial widening of the spectrum is mainly from phase self-modulation (SPM). The optical wave breaking (OWB) begins 
to show up when the input pulse energy is higher than $0.5 \mathrm{~nJ}$. The spectral width will increase with the increase of the input pulse energy.

For input pulse energy of $1.5 \mathrm{~nJ}$, the SC generation is expected with bandwidth of $1040 \mathrm{~nm}$ around the pumping wavelength in the range $1252-2292 \mathrm{~nm}$ of wavelengths after propagating about $20 \mathrm{~cm}$ inside the PCF as shown in Fig. 5(b).


Fig. 6. Numerical calculations of the spectral (a) and temporal evolution (b) - (c) of the pulse along the fiber in $\mathrm{CS}_{2}$ - filled core PCF.

Meanwhile, Fig. 6 depicts the spectral and temporal evolution of the pulse along the propagation distance with input pulse energy $1.5 \mathrm{~nJ}$. In this case, the location of pump wavelength is in the normal dispersion region, after the initial widening of the spectrum due to phase selfmodulation, which is characterized by temporal spectrogram in S-shape as Fig. 6 (b). Next, we observe a further widening of the spectrum in the short wavelength range due to OWB. As shown in Fig. 6 (a), OWB firstly occurs in the trailing edge of the pulse at $2.0 \mathrm{~cm}$ of propagation and generates the new wavelength band around $1.20 \mu \mathrm{m}$. After around $2.0 \mathrm{~cm}$ of propagation, the 
broad spectrum is asymmetric with a larger broadening on the long wavelength side as the propagation distance increase. On the leading edge, OWB occurs only after $14 \mathrm{~cm}$ of propagation and creates a new wavelength band around $2.0 \mu \mathrm{m}$. For further propagation, the energy of the pulse is redistributed from the central area to the edges resulting in the further flat on the wings of the output spectrum. It is clear that because different frequency components usually have different velocities, time delay between different frequencies becomes larger with a longer propagation as shown in Fig. 6 (c).

Table 3. The comparison between the properties of proposed PCFs and some other $\mathrm{CS}_{2}$ core PCFs

\begin{tabular}{lcccccc}
\hline Type of fibers & Pump wavelength Regime Pulse length Pulse Energy & SC range (nm) & Refs. \\
\hline $\mathrm{CS}_{2}$-core PCF & $1.55 \mu \mathrm{m}$ & Normal & $90 \mathrm{fs}$ & $1.5 \mathrm{~nJ}$ & $1252-2292$ & This work \\
$\mathrm{CS}_{2}$-core PCF & $1.56 \mu \mathrm{m}$ & Normal & $180 \mathrm{fs}$ & $50 \mathrm{~mW}$ & $1460-2100$ & {$[20]$} \\
$\mathrm{CS}_{2}$-core PCF & $1.55 \mu \mathrm{m}$ & Normal & $200 \mathrm{fs}$ & $0.4 \mathrm{~nJ}$ & $1000-2000$ & {$[34]$} \\
$\mathrm{CS}_{2}$-core PCF & $1.55 \mu \mathrm{m}$ & Normal & $500 \mathrm{fs}$ & $1.0 \mathrm{~nJ}$ & $1355-2110$ & {$[35]$} \\
$\mathrm{CS}_{2}$-core PCF & $1.55 \mu \mathrm{m}$ & Normal & $450 \mathrm{fs}$ & $45 \mathrm{~mW}$ & $1000-2200$ & {$[36]$} \\
\hline
\end{tabular}

Table 3 shows the comparison between the properties of the proposed PCF and those obtained in some previous works. Here the lasers emitting wavelength of around $1.55 \mu \mathrm{m}$ were used as pump sources. It can be seen that we obtained a SC spectra range with a similar bandwidth as those obtained in previous works but higher coherence and lower noise.

\section{CONCLUSION}

In this paper, we have presented a numerical simulation on optimum struture of a PCF made of fused silica with $\mathrm{CS}_{2}$-filled-core for obtaining all normal dispersion charactericties. The large scope optimization process of the PCF structure, due to the modifications of their micro-structured geometries, has been carried out in order to achieve the flat dispersion, and the generation of SC in the whole normal dispersion region with pumping at $1.55 \mu \mathrm{m}$. According to the conducted simulations, optimized fibre with the lattice constant $\Lambda=\mu \mathrm{m}$, filling factor $f=0.3$ exhibited an all-normal dispersion and its peak equals $-8.1 \mathrm{ps} / \mathrm{nm} / \mathrm{km}$ at $1.55 \mu \mathrm{m}$.

Our numerically simulated results demonstrated that in $\mathrm{CS}_{2}$ filled-core optimal PCF structure, the SC with a broadened spectral bandwidth of $1252 \mathrm{~nm}$ to $2292 \mathrm{~nm}$ was generated by a pump pulse with a central wavelength of $1.55 \mu \mathrm{m}, 90 \mathrm{fs}$ duration and energy of $1.5 \mathrm{~nJ}$. Further increase in the spectral width can be expected if we increase input pulse energy. Due to the higher nonlinearity of $\mathrm{CS}_{2}$ than that of fused silica, lower power of input pulses is required than in the case of silica PCFs [23]. Those fibers would be good candidates for all-fiber SC sources as cost-effective alternatives to glass core fibers.

\section{ACKNOWLEDGEMENT}

This work was supported by the project 796/2019/ HĐKHCN-ĐTKHCN. 


\section{REFERENCES}

[1] J. C. Knight, Nature. 424 (2003) 847-851.

[2] R. Buczynski, Acta Physica Polonica A. 106(2) (2004) 141.

[3] T. A. Birks, J. C. Knight, P. S. J. Russell, Optics Letters 2(13) (1997) 961.

[4] Z. Hui, D. Hou, Y. Zhang, S. Wei, and J. Xu, Fiber and Integrated Optics 38(2) (2019) 91.

[5] A. Bala, K. R. Chowdhury, M. B. Mia, and M. Faisal, Appl. Opt. 56 (2017) 7256.

[6] J. Lægsgaard, P.J. Roberts, and M. Bache, Optical and Quantum Electronics, 39(12-13) (2007) 995.

[7] J. M. Hsu, Optics Communications 361 (2016) 104.

[8] M. Guillon, K. Dholakia, and D. McGloin, Opt. Express 16 (2008) 7655.

[9] H. Tu and S. A. Boppart, Laser Photonics Rev.7(5) (2013) 628.

[10] W. J. Ling, K. Li. and Y. Y. Zuo, Applied Mechanics and Materials, 302 (2013) 194.

[11] H. Liu, Y. Yu, W. Song, Q. Jiang, and F. Pang, Opto-Electronic Advances, 2(2) (2019) 1800201.

[12] J. M. Dudley, G. Genty, and S. Coen, Rev. Modern Phys. 78(4) (2006) 1135.

[13] A. M. Heidt, J. Opt. Soc. Am. B 27 (2010) 550.

[14] L. E. Hooper, P. J. Mosley, A. C. Muir, W. J. Wadsworth, and J. C. Knight, Opt. Express, 19 (6) (2011) 4902.

[15] A. V. Gorbach, D. V. Skryabin, J. M. Stone, and J. C. Knight, Opt. Express 14(21) (2006) 9854.

[16] A. V. Husakou, and J. Herrmann, Phys. Rev. Lett. 87(20) (2001) 203901.

[17] J. K. Ranka, R.S. Windeler, and A.J. Stentz, Opt. Lett. 25 (1) (2000) 25.

[18] Z. X. Jia, C. F. Yao, S. J. Jia, F. Wang, S. B. Wang, Z. P. Zhao, M. S. Liao, G. S. Qin, L. L. Hu, Y. Ohishi, and W. P. Qin, Laser Phys. Lett. 15(2) (2018) 025102.

[19] S. Dai, Y. Wang, X. Peng, P. Zhang, X. Wang, and Y. Xu, Appl. Sci. 8(5) (2018) 7071.

[20] D. Churin, T. N. Nguyen, K. Kieu, R. A. Norwood, and N. Peyghambarian, Opt. Mater. Express. 3(9) (2013) 1358.

[21] L. C. Van, A. Anuszkiewicz, A. Ramaniuk, R. Kasztelanic, K. D. Xuan, V. C. Long, M. Trippenbach and R. Buczyński, J. Opt. 19(12) (2017) 125604.

[22] Q. H. Dinh, J. Pniewski, H. L. Van, A. Ramaniuk, V. C. Long, K. Borzycki, K. D. Xuan, M. Klimczak and R. Buczyński, Appl. Opt. 57(14) (2018) 3738.

[23] H. V. Le, V. C. Long, H. T. Nguyen, A. M. Nguyen, R. Kasztelanic, and R. Buczyński, Laser Phys. 28(11) (2018) 115106.

[24] C. V. Lanh, V. T. Hoang, V. C. Long, K. Borzycki, K. D. Xuan, V. T. Quoc, M. Trippenbach, R. Buczyński, and J. Pniewski, Laser Phys. 29(7) (2019) 075107.

[25] L. C. Van, V. T. Hoang, V. C. Long, K. Borzycki, K. D. Xuan, V. T. Quoc, M. Trippenbach, R. Buczyński, and J. Pniewski, Laser Phys. 30(3) (2020) 035105.

[26] P. S. Maji and P. R. Chaudhuri, Optik 125 (2014) 5986.

[27] N. Munera and R. A. Herrera, Opt. Commun. 368 (2016) 185.

[28] T. Kato, Y. Suetsugu, M. Takagi, E. Sasaoka, and M. Nishimura, Opt. Lett. 20(9) (1995) 988.

[29] E. K. Plyler, and C. J. Humphreys, J. Res. Nat. Bur. Standards 39 (1947) 59.

[30] Lumerical Solutions, Inc., http://www.lumerical.com.

[31] G. Stepniewski, R. Kasztelanic, D. Pysz, R. Stepien, M. Klimczak, R. Buczynski, Opt. Mater. Express 6(8) (2016) 2689.

[32] S. Kedenburg, M. Vieweg, T. Gissibl and H. Giessen, Opt. Mater. Express 2(11) (2012) 1588.

[33] G. P. Agrawal, Nonlinear fiber optics, 5th edn, Oxford: Academic Press, 2013.

[34] R. Zhang, J. Teipel and H. Giessen, Opt. Express 14(15) (2006) 6800.

[35] M. Chemnit, C. Gaida, M. Gebhardt, F. Stutzki J. Kobelke, A. Tunnermann, J. Limpert and M. A. Schmidt Opt. Express 26 (3) (2018) 3221.

[36] Z. Kang, F. Xu, J. Yuan, S. Member, F. Li , B. Yan, X. Zhou, Q. Wu , K. Wang, X. Sang, K. Long, S. Member and C. Yu IEEE J. Quantum Electron. 55(2) (2019) 1. 